\title{
Estratificación vertical de briófitos epífitos encontrados en Quercus humboldtii (Fagaceae) de Boyacá, Colombia
}

\section{Jorge Enrique Gil Novoa \& María Eugenia Morales Puentes}

Grupo Sistemática Biológica, Herbario UPTC, Escuela de Biología, Universidad Pedagógica y Tecnológica de Colombia. Av. Central Norte, Tunja, Colombia; jorge.gil@uptc.edu.co, maria.morales@uptc.edu.co

\author{
Recibido 08-IV-2013. Corregido 10-XI-2013. Aceptado 10-XII-2013.
}

\begin{abstract}
Vertical stratification of epiphytic bryophytes found on Quercus humboldtii (Fagaceae) from Boyacá, Colombia. Oak forest represents an ecologically important plant formation in Colombia, partly due to the large amount of epiphyte flora that harbors, especially non-vascular plants which have been poorly studied in Colombia. One of the biggest oak forests in the country is the one found in the Parque Natural Municipal "Robledales de Tipacoque" (PNMRT), in Boyacá, municipality of Tipacoque. The epiphyte bryoflora was evaluated in $Q$. humboldtii, using canopy climbing techniques and dividing the trees ( 25 individuals sampled) each into five layers (base, trunk, inner canopy, middle and outer canopy). A total of 365 samples were collected: $29 \%$ liveworts and $71 \%$ mosses. Hepatics represented 10 families, 16 genera and 26 species; mosses 11 families, 26 genera and 49 species. Considering the tree layers, the most diverse one was the base with 51 species, followed by the trunk with 43 ; in the canopy, the inner canopy was found the most diverse with 28 species, and was followed by the middle canopy with 18 , and the outer canopy with 15 . A species similarity index shows that the trunk and the middle canopy were the most closely related (0.42) in terms of species composition. The results showed that non-vascular plants were mostly found in lower layers (base and trunk), where the diversity was greater, richer (species number), and this might be caused by the microclimatic conditions in these places, such as higher humidity, lower light intensity and shade. Thus, some species are considered shade epiphytes (ombrophiles) because they are unique to these areas, like Bazzania gracilis and Taxilejeunea pterigonia, among others. Likewise, Jungermannia sp. is considered a sun epiphyte (heliophilous) because it is found only in the outer canopy. We concluded that $Q$. humboldtii could be considered as a potential host for the conservation of non-vascular epiphytes in Colombian forests. Rev. Biol. Trop. 62 (2): 719-727. Epub 2014 June 01.
\end{abstract}

Key words: Andes, Colombia, epiphytes bryophytes, liverworts, mosses, Quercus, vertical stratification.

Quercus humboldtii, es una especie endémica de Colombia y Panamá, de origen holártico ( 300 000 años) (Van der Hammen, 2000), y es la única especie de este género presente en el norte de Sur América. La formación vegetal donde se encuentra esta especie, y domina es conocida como robledal, entre los 1100 y $3500 \mathrm{~m}$ de altitud. Un estudio realizado por Marín-Corba \& Betancur (2007) menciona que la riqueza de especies es similar en los robledales y los bosques andinos tropicales a la misma altitud.

Las características más sobresalientes de los bosques andinos tropicales son, la elevada humedad y la reducida iluminación, condiciones que permiten la abundancia de epífitas (Wolf, 2003; Gradstein et al., 1996; Aguirre-C., 2008a); por lo anterior, es importante entender los mecanismos de relación epífito-hospedero para comprender la distribución espacial de estos organismos en los bosques. Sin embargo, existe evidencia que tal distribución depende de factores como la textura del hospedero, la porosidad de su corteza, el $\mathrm{pH}$, la cantidad de luz y la humedad (Alzate \& Cardona, 2000; Mota, Ter Steege, Cornelissen \& Gradstein, 2009).

Las epífitas en general tienen preferencias en su distribución que han sido difíciles 
de estimar. Sin embargo, Mota et al. (2009) mencionan que la distribución vertical de briófitos en bosques tropicales no sigue siempre el mismo patrón, dado que depende de variables ambientales, aunque, algunas especies epífitas prefieren las zonas altas de los árboles (dosel), posiblemente influenciados por condiciones como la temperatura, la luz y la velocidad del viento, las cuales, son fuertes en dichas zonas del árbol, independientemente del tipo de bosque o la especie de forófito en la cual se encuentre.

En Colombia, se resaltan trabajos en plantas vasculares epífitas como los de Linares (1999), Arévalo \& Betancur (2007), Isaza, Betancur \& Stévez (2004), Jácome, Galeano, Amaya \& Mora (2004); y son escasos los estudios sobre la distribución vertical de epífitas no vasculares, conociéndose únicamente para la cordillera Central colombiana, el trabajo de Wolf (1995) en briófitos y líquenes, y para la cordillera Oriental, el de Simijaca (2011) en líquenes. Por lo anterior, esta investigación evaluó la distribución vertical de los briófitos en árboles de $Q$. humboldtii, en el Parque Natural Municipal Robledales de Tipacoque, en Tipacoque (Boyacá - Colombia), siendo este un estudio pionero para el país.

\section{MATERIALES Y MÉTODOS}

Área de estudio: El Parque Natural Municipal Robledales de Tipacoque (PNMRT) se encuentra ubicado al norte del departamento de Boyacá, en la zona rural del municipio de Tipacoque. El área de influencia está representada por 1159 ha, entre los 2800 a $3300 \mathrm{~m},\left(6^{\circ} 23^{\prime}\right.$ $\mathrm{N} ; 72^{\circ} 42^{\prime \prime}$ O') y precipitaciones promedio de $1081.85 \mathrm{~mm} /$ año, y una temperatura promedio de $17.4^{\circ} \mathrm{C}$ (Fundación Natura Colombia, 2007).

Selección de forófitos: Se delimitaron transectos lineales de $100 \mathrm{~m}$, dentro de cada uno, se marcaron cuatro árboles maduros (fustes gruesos y ramas resistentes, Pinzón \& Linares, 2006) de $Q$. humboldtii, de tal manera que permitieran su ascenso mediante técnicas de escalada simple (Perry, 1978). El número de forófitos se siguió bajo el criterio de Gradstein, Nadkarni, Krömer, Holz \& Nözke (2003). Cada forófito se dividió en cinco niveles o estratos verticales (Johansson, 1974); lo anterior, considerando la recolección de briófitos y las estimaciones de diversidad. Se estimó la cobertura de cada una de las especies con ayuda de una plantilla de acetato cuadriculada de $400 \mathrm{~cm}^{2}$ (Iwatzuki, 1960). Para el caso de las ramas externas, la plantilla se modificó a un área de $100 \mathrm{~cm}^{2}$ (Wolf, 1993).

El proceso de identificación del material se llevó a cabo en el herbario UPTC, mediante la utilización de claves especializadas (Churchill \& Linares, 1995; Gradstein, 1994; Gradstein, et al., 2001; Heinrichs, 2002; Uribe \& Aguirre, 1997; Sharp, Crum \& Eckel, 1994), entre otros. Luego del proceso curadurial, el material se incluyó en la colección de referencia (Herbario UPTC). Se determinó la diversidad mediante los índices de Shannon-Wiener y Margalef (Moreno, 2001); para analizar la relación entre la distribución de las especies, se realizó un análisis de Escalamiento Multidimensional No-Métrico (NMS) utilizando el programa estadístico PAST (Versión 2.05). Se realizó un análisis de varianza (ANOVA) multifactorial para determinar diferencias significativas en el número de especies en las zonas del Parque, y entre los estratos, utilizando el programa STATGRAPHICS (versión 5.1).

\section{RESULTADOS}

En los 203 levantamientos realizados se recolectaron 365 muestras, de las cuales, el 29\% pertenecieron a hepáticas y el $71 \%$ a musgos.

Musgos: Se encontró un total de 259 muestras, en 11 familias, 26 géneros y 49 especies; los musgos acrocárpicos fueron los más abundantes con el $65.54 \%$ de las especies, mientras que el $33.46 \%$ correspondió a especies pleurocárpicas. La familia con mayor número de especies fue Dicranaceae con 15, seguida de Sematophyllaceae con 13 y Calymperaceae con nueve. Los géneros más representativos fueron: Campylopus con seis especies, y Syrrhopodon 
con cinco, seguidos de Calymperes y Sematophyllum cada uno con cuatro taxones.

Hepáticas: Se encontró un total de 96 ejemplares, en 10 familias, 16 géneros y 26 especies, donde la forma de crecimiento foliosa fue la dominante con el $84.37 \%$, mientras que el $15.63 \%$ restante fueron hepáticas talosas. Plagiochilaceae y Lepidoziaceae fueron las familias más representativas con cinco especies cada una, seguidas de Geocalycaceae, Jungermanniaceae y Lejeuneaceae con tres taxones. Los géneros con mayor diversidad son Plagiochila (cinco especies), Heteroscyphus, Jungermannia y Metzgeria (dos especies cada uno), los demás géneros presentaron una especie cada uno. Los taxones con mayor riqueza fueron: Lepidozia brasiliensis con 19 registros, Anoplolejeunea conferta (13) y Herbertus acanthelius (nueve); mientras que las especies que registraron las mayores coberturas fueron: L. brasiliensis con $1484 \mathrm{~cm}^{2}, H$. acanthelius $\left(704 \mathrm{~cm}^{2}\right)$ y Tylimanthus diversifolius $\left(448 \mathrm{~cm}^{2}\right)$.

Se realizó un análisis de similitud entre estratos, con dos tendencias; la primera corresponde a la relación entre el tronco y el dosel interno del árbol con el $41 \%$ de especies compartidas, y a su vez, se establece una relación de similitud del $30 \%$ con la base del árbol, y la segunda tendencia (35\%) es la relación entre el dosel medio y el dosel externo del árbol (Fig. 1).

Diversidad: Los análisis de diversidad (Shannon-Wiener), muestran que las zonas dos y cinco presentaron distribución de los organismos de una manera más uniforme que con las zonas restantes, con valores de 3.66 y 3.67 ; mientras que la zona tres es la que presenta mayores diferencias (3.42). De igual manera, a través del índice de Simpson, se muestra que, la zona tres presenta el menor valor de dominancia, siendo éste el transecto con mayor riqueza específica (0.96); caso contrario, lo presenta la zona dos con el mayor valor de dominancia (0.97), e indica una riqueza específica menor que en las demás zonas. Según el cálculo del índice de Margalef, la zona cinco fue la más diversa (10.23), seguido de la zona dos (10.19); por el contrario, la zona tres presentó los menores valores de diversidad (8.52).

A través del índice de Shannon-Wiener los estratos tronco y dosel externo, fueron los estratos con una distribución más uniforme (3.60 y 3.62, respectivamente). Por el contrario, el estrato con mayor disimilitud fue la base del árbol (estrato tres) al presentar un valor de 3.42. En cuanto la dominancia (índice de Simpson), el dosel medio fue el estrato con mayor riqueza específica y el menor valor de dominancia. Caso contrario presentó la base del árbol, con un valor más alto (0.97), lo que indica, que es el estrato con menor riqueza específica. Mientras que el estrato con mayor diversidad fue el dosel externo (10.23), seguido del tronco (10.19), contrastando con el dosel interno, estrato con el menor valor de diversidad (8.52).

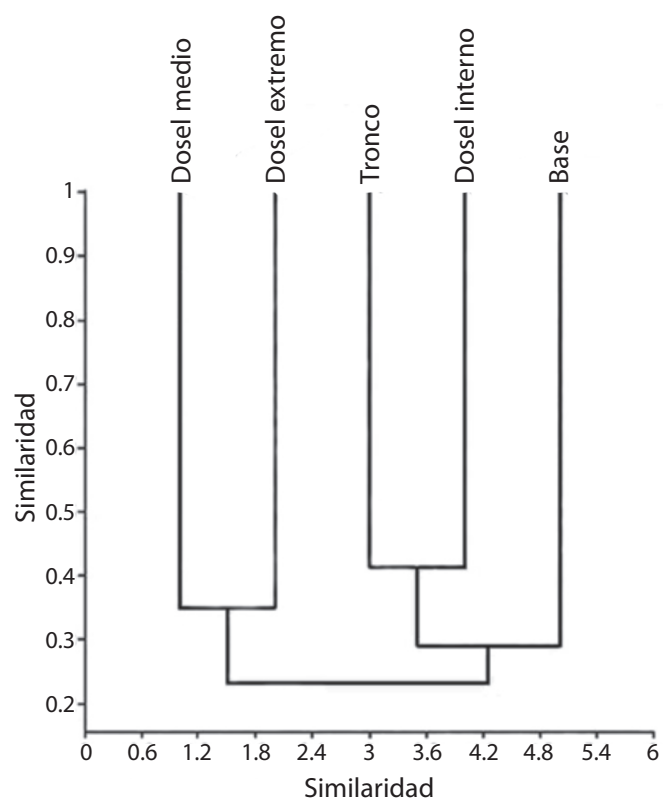

Fig. 1. Análisis de clúster que muestra la relación de similitud entre los diferentes estratos evaluados en árboles de $Q$. humboldtii para el Parque Natural Municipal "Robledales de Tipacoque".

Fig. 1. Cluster analysis showing the relationship of similarity between the different layers evaluated in $Q$. Humboldtii trees, Parque Natural Municipal "Robledales de Tipacoque". 
El análisis de Escalamiento Multidimensional No-Métrico (NMS) (Fig. 2), mostró similitud entre la base del tronco, reflejándose esta relación entre las zonas dos-cinco y cuatro-tres. Para el caso del tronco, se identificó una fuerte relación entre todas las zonas, con muy pocas diferencias, especialmente entre las zonas tres-cuatro. Por el contrario, las mayores diferencias entre zonas las presenta el dosel interno, donde las relaciones para este estrato con todas las zonas son muy dispersas, principalmente de las zonas uno y dos con las demás.

Distribución de las especies: De acuerdo con los resultados del ANOVA, no existen diferencias significativas entre las zonas $(\mathrm{p}>0.05)$; por el contario, los estratos comparados reflejan un efecto significativo $(\mathrm{p}<0.05)$. Con un $99 \%$ de confiabilidad, las pruebas a posteriori de Tukey y Scheffe distinguen dos grupos significativamente diferentes: el primero de ellos conformado por los tres estratos de dosel (interno, medio y externo), el segundo conformado por el tronco y la base del árbol.

\section{DISCUSIÓN}

Se registraron 26 especies de hepáticas, que representan el $3.12 \%$ del total de las especies presentes en el país (Uribe \& Gradstein, 1999); en musgos se encontraron 48 especies (4.9\% de las registradas para el país) (AguirreC., 2008b), lo cual significa, una baja diversidad. Sin embargo, al comparar este trabajo con otros similares, como los de Holz \& Gradstein (2005a, 2005b), realizado para bosques primarios de Quercus en Costa Rica, se observa que para Tipacoque existe mayor número de especies de musgos epífitos encontrados en el mismo género de árbol hospedero (48 en Tipacoque/41 en Costa Rica). El caso contrario, sucedió con las hepáticas, con una diferencia de 41 especies para los dos lugares (26/67 respectivamente). Los resultados registrados en

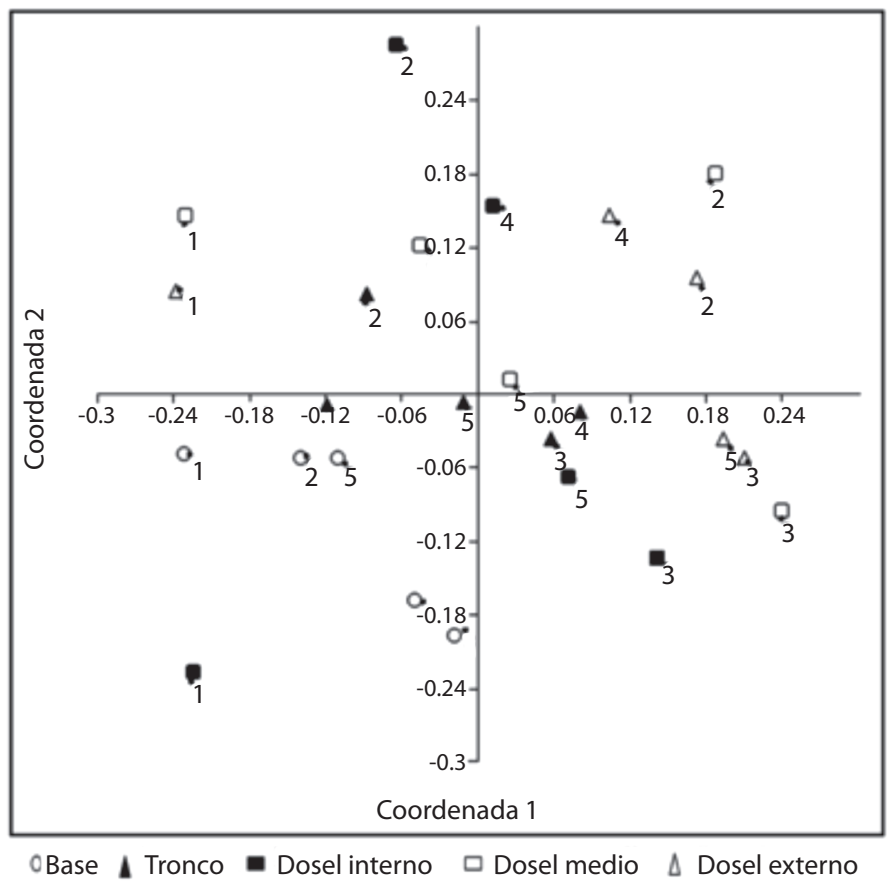

Fig. 2. Análisis NMS con la relación entre cada una de las cinco zonas evaluadas (números) y los cinco estratos en los árboles (símbolos). Cada uno de los números representa un estrato.

Fig. 2. NMS analyzes with the relationship between each of the five areas assessed (numbers) and the five layers in the trees (symbols). Each number represents a layer. 
cada uno de las zonas muestreadas, evidencian que el "Alto El Frailejonal" y el "Alto de Las Águilas" son las zonas con mayor diversidad y similitud de especies briófitas. Lo anterior, probablemente se deba a la homogeneidad de la vegetación vascular, con árboles de porte similar en un mismo rango altitudinal. Estos resultados concuerdan con la propuesta de Wolf (2003) y Sillet \& Antonie (2004), donde la humedad, la incidencia de luz, fuertes vientos y las características microambientales semejantes en dos lugares, pueden influir en la distribución de estas plantas.

En el "Alto El Encenillo" y "Alto de La Paja", se registró la menor diversidad de briófitos de las cinco zonas, al identificarse 27 especies en cada una de ellas, datos que contrastan con el mayor grado de conservación de estos parches de bosque. Según Holz \& Gradstein (2005b), la diversidad disminuye en los bosques secundarios, debido a la modificación de la vegetación, pero resaltan que en ocasiones éstos bosques pueden presentar el efecto contrario, con un aumento en la diversidad de plantas no vasculares epífitas, acción causada por el ecotono producido durante la interacción de los dos ecosistemas, donde existe migración de especies por parte de las dos formaciones vegetales; este último caso, puede ser la explicación para que las dos zonas menos afectadas por intervención humana, sean al mismo tiempo, las de más baja diversidad.

El análisis de correlación de las cinco zonas estudiadas, permite evidenciar la presencia de especies compartidas, con dos tendencias similares a las mencionadas anteriormente, en cuanto a las zonas de mayor diversidad del Parque: la primera tendencia, reúne a las zonas "El Encerrado" y "Alto El Encenillo que comparten cerca del $73 \%$ de especies; esta relación se puede dar por la similitud en las dos formaciones vegetales, y con alturas similares de los forófitos (entre 14-18m), y como la vegetación acompañante, donde comparten elementos de Cunoniaceae, Melastomataceae y Clusiaceae. También, el análisis clúster muestra otro grupo conformado por las zonas "Alto de Las Águilas" y "Alto El Frailejonal", que comparten el
$47 \%$ de las especies, aunque son las zonas con el índice de similitud más bajo. Del total de especies recolectadas, las hepáticas $A$. conferta y L. brasiliensis y los musgos Campylopus sp.1, Leptodontium sp., S. cryptocarpus y Thuidium peruvianum se pueden considerar con una mayor amplitud de distribución, y se encuentran en las cinco zonas evaluadas.

Del total de especies recolectadas (74), el $71.62 \%$ se presentan en la base del árbol, situación que permite definir a este estrato como el preferido por los briófitos. Mota et al. (2009) explican lo anterior, debido las condiciones microambientales como la baja cantidad de luz y la alta humedad para este estrato. Así mismo, se encontraron 53 especies, de las cuales, Calymperes sp2, Plagiochila aerea y P. borya$n a$ son propias de este estrato. Esta preferencia se debe a que los lugares de sombra $(\sim 85 \%$ de cobertura), son estratos donde las especies de briófitos necesitan mucho más tiempo para establecerse y desarrollar las adaptaciones para estos microambientes (Holz \& Gradstein, 2005). Esto indica que algunas de las especies que se encuentran en la base de los árboles, también se pueden encontrar en otros estratos, probablemente producto de la migración de propágulos o esporas (Wolf, 1993).

Después de la base del árbol, el tronco es el segundo estrato con mayor diversidad al registrar 43 especies, de las cuales, seis son exclusivas de este estrato, lo que indica que estas especies son bastante sensibles a los disturbios ambientales en los bosques (Vanderpoorten, Engels \& Sotiaux, 2004). Se resalta que $P$. flexilis, se encontró solamente para éste estrato, sin embargo, es un musgo con un rango de distribución bastante amplio (700-4000m) y que crece, tanto en troncos como en dosel de árboles (Gradstein, 2001); así, características morfológicas como el crecimiento pendular, que le permite retener mayor cantidad de agua, y favorece tanto su crecimiento como su fácil dispersión (Romero, Puts \& Kitajima, 2006).

En la parte interna del dosel se registra la mayor diversidad, con 28 especies, seguida de la zona media (18) y las ramas externas (15). Así mismo, en la parte interna del dosel se 
observaron tres especies exclusivas para este estrato: Heteroscyphus elliotii, Metzgeria aff. neotropica y M. longiseta, y se pueden definir como epífitas especialistas de sombra (Acebey, Gradstein \& Krömer, 2003). Para el caso de las ramas externas, sólo se registró Jungermannia sp., denominada epífita de sol, debido a su preferencia por este estrato. Estos resultados indican que en bosques de $Q$. humboldtii, la diversidad de briófitos con preferencia por ambientes sombríos es mayor, que los de preferencia por incidencia de luz directa. Se observa que en todos los estratos dominaron los musgos, lo que sugiere mayor tolerancia de éstos a lo largo de la variabilidad microclimática de los estratos en el árbol con respecto a las hepáticas; además de otras características, como es el caso de la corteza, y específicamente las fisuras, que permiten la acumulación de nutrientes y agua, y que sirven de anclaje para las epífitas (Linares, 1999). Algunas especies de briófitas como Campylopus sp.1., Sematophyllum napoanum, Syrrhopodon aff. xanthophyllus y S. cryptocarpus, se consideran de amplia distribución, al encontrarse en todos los estratos. Estos géneros coinciden con lo presentado por AguirreC. (2008a), quien menciona a Campylopus, Syrrhopodon y Sematophyllum, como los grupos con mayor preferencia por el sustrato cortícola en el sistema cordillerano colombiano.

El análisis de clúster que relaciona la similitud (índice de Jaccard) entre los diferentes estratos, muestran que la mayor relación entre estratos, la presentan el tronco y el dosel interno (Fig. 2) con cerca del 0.42. Esta correlación está dada por el número de especies que comparten los dos estratos, probablemente por el efecto de sombra que genera el dosel, tanto del mismo árbol, como de los árboles vecinos, lo que conlleva a la disminución en la velocidad de los vientos, baja incidencia de luz y alta humedad (Mota et al, 2009). Otra relación, a pesar de los valores bajos (0.35), está dada por los estratos dosel medio y externo del árbol, donde las condiciones microclimáticas (humedad, temperatura, velocidad del vientos e incidencia de luz) pueden ser similares, por tanto, es probable que el número de especies que comparten los dos estratos sea similar.

El estrato base de los árboles, aunque presentó mayor diversidad, fue el nivel con menor similitud entre zonas, debido, tal vez, al ecotono que se presenta en esta parte del árbol, al interactuar con las raíces aflorantes y directamente con el suelo, donde algunas variables como la humedad y la temperatura pueden cambiar drásticamente. Al comparar los resultados obtenidos en el estrato base, con los datos presentados por Vargas (2011), en la misma área de estudio, se evidencia que en este estudio la diversidad de hepáticas fue menor (18 vs. 21, Vargas, 2011); lo anterior, se debe a que esta investigación se desarrolla solo sobre Q. humboldtii. También Porras (2011) realizó un inventario de musgos en raíces aflorantes de roble en Tipacoque, donde la diversidad fue menor que la presentada en este trabajo, lo que indica que la base del forófito es el estrato con mejores condiciones para el desarrollo de la flora no vascular. Algunas especies tuvieron preferencia por zonas del árbol con menor incidencia de luz y, se denominan epífitas de sombra (Richards, 1984) como, las hepáticas: Heterocyphus elliottii, Metzgeria aff. neotropica, M. filicina y Symphyogyna brongniartii (dosel interno), y los musgos: A. bogotense y $T$. peruvianum (base), lo que sugiere, que son especies muy sensibles a los cambios ambientales, ya que, tanto en la base como en el dosel interno, encuentra protección y por tanto, menor incidencia de efectos negativos causados por la intervención del bosque (Martins, 2009).

El análisis de ANOVA, muestra que no existen diferencias significativas entre las cinco zonas evaluadas, lo que indica que la composición en cuanto al número de especies, es similar en toda la zona de estudio; sin embargo, las mínimas diferencias que se muestran son variaciones entre 28 y 32 especies entre zonas. Contrario a lo anterior, el análisis permite evidenciar que existen diferencias significativas entre los cinco estratos del árbol, y se aplicaron las pruebas post-hoc de Tukey y Scheffe. Los análisis de estas pruebas coinciden en que las 
zonas de la base y del tronco son muy similares en cuanto a número de especies (53 y 42, respectivamente), y comparten algunas especies como A. proligera, S. napoanum y L. brasiliensis, entre otras; de igual manera, estos taxones son las que presentan la mayor riqueza. Según Vann Lerdam, Zagt \& Veenklass, (1990), esta relación se puede presentar por similitud en variables como la cantidad de luz y velocidad del viento, principalmente, y la disponibilidad de nutrientes, la cual, sería mayor para estos estratos. Aunque en este trabajo estas variables no fueron consideradas, los datos permiten inferir la probabilidad, de que, estas variables sean similares.

\section{AGRADECIMIENTOS}

A la Universidad Pedagógica y Tecnológica de Colombia, Herbario UPTC y Grupo Sistemática Biológica. A la administración y a los habitantes del municipio de Tipacoque. A Inés Sastre de Jesús (Universidad de Puerto Rico), Edgar Linares (Universidad Nacional de Colombia), Paulo Câmara (Universidad de Brasilia), Robert Gradstein (Muséum National d'Histoire Naturelle, Paris) y María E. Reinier (University of Göttingen). A Lucía Vargas, Stefanny Porras, Lorena Hernández y Diego Simijaca (Universidad Pedagógica y Tecnológica de Colombia).

\section{RESUMEN}

Los bosques de roble representan una formación vegetal de importancia ecológica, debido a la cantidad de plantas epífitas que pueden llegar a albergar; estas han sido muy poco estudiadas en Colombia, especialmente las no vasculares. Para el país, uno de los robledales de mayor extensión es el Parque Natural Municipal "Robledaes de Tipacoque" (PNMRT), que se encuentra ubicado en Boyacá, en el municipio de Tipacoque. Se evaluó la brioflora epífita en Quercus humboldtii, mediante técnicas de ascenso a dosel y división de cada uno de los árboles (25 individuos muestreados) en cinco estratos cada uno (base, tronco, dosel interno, dosel medio y dosel externo). Se recolectaron en total 365 muestras, $29 \%$ pertenecen a hepáticas y $71 \%$ a musgos. Las hepáticas se distribuyeron en 10 familias, 16 géneros y 26 especies, mientras que los musgos se distribuyeron en 11 familias, 26 géneros y 49 especies. La base del árbol es el estrato con mayor diversidad de especies (51), seguida del tronco (43), mientras que en el dosel, la parte interna fue la más diversa con 28 especies, seguida del dosel medio con 18 y el dosel externo con 15. Los análisis de similitud muestran una baja relación entre los estratos, donde la mayor afinidad esta entre el tronco y el dosel medio con el 0.42 . Los resultados muestran que las plantas no vasculares prefieren los estratos inferiores (base y tronco), donde la diversidad es mayor, debido a las condiciones microclimáticas que éstas zonas presentan, como mayor humedad, menor intensidad lumínica y sombra; además, algunas especies se consideran como epífitas de sombra (hombrófilas) al ser exclusivas de éstas zonas, como Bazzania gracilis y Taxilejeunea pterigonia, entre otras. El caso contrario, Jungermannia sp. se considera epífita de sol (heliófila) al encontrarse únicamente en el dosel externo. Se concluye que $Q$. humboldtii puede ser un hospedero potencial para la conservación de epífitas no vasculares en bosques colombianos.

Palabras clave: Andes, estratificación vertical, briófitos epífitos, hepáticas, musgos, forófito, Quercus.

\section{REFERENCIAS}

Acebey, A., Gradstein, S. R., \& Krömer, T. (2003). Species richness and habitat diversification of bryophytes in submontane rain forest and fallows of Bolivia. Journal of Tropical Ecology, 19(1), 9-18.

Aguirre-C, J. (2008a). Diversidad y riqueza de musgos y líquenes en Colombia-Generalidades y metodologíaIn O. Rangel-Ch (Ed.). Colombia diversidad biótica VI: Riqueza y diversidad de los musgos y líquenes en Colombia (pp. 1-17). Bogotá: Universidad Nacional de Colombia, Facultad de Ciencias, Instituto de Ciencias Naturales.

Aguirre-C, J. (2008b). Diversidad y riqueza de los musgos en la región natural andina o sistema cordillerano. In O. Rangel-Ch (Ed.). Colombia diversidad biótica VI: Riqueza y diversidad de los musgos y líquenes en Colombia (pp. 19-54). Bogotá: Universidad Nacional de Colombia, Facultad de Ciencias, Instituto de Ciencias Naturales.

Alzate, F. \& Cardona, F. (2000). Patrones de distribución de epífitas vasculares en "robledales". Revista de la Facultad Nacional de Ciencias Agropecuarias, 58(1), 969-983.

Arévalo, R. \& Betancur, J. (2004). Diversidad de epífitas vasculares en cuatro bosques de la zona suroriental de la serranía de Chiribiquete, Guayana colombiana. Caldasia, 26(2), 359-380.

Churchill, S. \& Linares, E. (1995). Prodromus Bryoloqiae Novo-Granatensis. Introducción a la flora de musgos de Colombia. Partes 1 y 2. Bogotá: Editorial Guadalupe Ltda. 
Fundación Natura Colombia. (2007). Diagnóstico socioambiental del PNM "Robledales de Tipacoque" y su área de influencia. Convenio 106-07.

Gradstein, S. R. (1994). Lejeuneaceae: Ptychantheae, Brachiolejeuneae. Flora Neotropica Monograph, 62, 1-217.

Gradstein, S. R., Hietz, P., Lücking, R., Lücking, A., Sipman, H., Vester, H., Wolf, J., \& Gardette, E. (1996). How to sample epiphytic diversity of tropical rain forest. Ecotropica, 2, 59-72.

Gradstein, S. R., Churchill, S., \& Salazar-Allen, N. (2001). Guide to the bryophytes of Tropical America. Memoirs of the New York Botanical Garden, 86, $1-577$.

Gradstein, S. R., Nadkarni, N., Krömer, T., Holz, I., \& Nözke, N. (2003). A protocol for rapid and representative sampling of vascular and non-vascular epiphyte diversity of tropical rain forest. Selbyana, 24(1), 105-111.

Heinrichs, J. (2002). A taxonomic revision of Plagiochila sect. Hylacoetes, sect. Adiantoideae and sect. Fuscoluteae in the Neotropics whith a preliminar subdivision of Neotropical Plagiochilaceae into nine lineages. Bryophytorum Bibliotheca, 58, 1-184.

Holz, I. \& Gradstein, S. R. (2005a). Cryptogamic epiphytes in primary and recovering upper montane oak forests of Costa Rica-species richness, community composition and ecology. Plant Ecology, 178, 89-109.

Holz, I. \& Gradstein, S. R. (2005b). Phytogeography of the bryophyte floras of oak forests and paramo of the cordillera de Talamanca, Costa Rica. Journal of Biogeography, 32, 1591-1609.

Isaza, C., Betancur, J., \& Estévez, J. V. (2004). Vertical distribution of bromeliads in a montane forest in the Eastern cordillera of the Colombian Andes. Selbyana, 25(1), 126-137.

Iwatsuki, Z. (1960). The epiphytic bryophyte communities in Japan. Journal Hattori Botanical Laboratory, 22, 159-339.

Jácome, J., Galeano, G., Amaya, M., \& Mora, M. (2004). Vertical distribution of epiphyte and hemiepihpytic Araceae in a tropical rain forest in Chocó, Colombia. Selbyana, 23, 118-123.

Johansson, D. R. (1974). Ecology of vascular epiphytes in West African rain forest. Acta Phytogeographyca Sueca, 59, 136.

Linares, E. L. (1999). Diversidad y distribución de las epífitas vasculares en un gradiente altitudinal en San Francisco, Cundinamarca. Revista de la Academia Colombiana de Ciencias, 23(Suplemento especial), 133-139.

Martins, A. C. (2009). Florística e ecologia das comunidades de briófitas em florestas de terra firme no Estado do Pará, Amazônia, Brasil (Tese de doutor em
Botânica). Instituto de Pesquisas Jardim Botânico do Rio Janeiro. Escola Nacional de Botânica Tropical.

Marín-Corba, C. \& Betancur, J. (1997). Estudio florístico en un robledal del Santuario de Flora y Fauna de Iguaque (Boyacá-Colombia). Revista de la Academia Colombiana de Ciencias Exactas, Físicas y Naturales, 21(80), 249-257.

Moreno, C. E. (2001). Métodos para medir la biodiversidad. MyT-Manuales y Tesis SEA, Zaragoza, 1.

Mota, S., Ter Steege, H., Cornelissen, J. C., \& Gradstein, S. R. (2009). Niche assembly of epiphytic bryophyte communities in the Guianas: a regional approach. Journal of Biogeography, 36, 2076-2084.

Perry, D. R. (1978). A method of access into the crowns of emergent and canopy trees. Biotropica, 10, 155-157.

Pinzón, M. \& Linares, E. (2006). Diversidad de líquenes y briófitos en la región subxerofítica de la Herrera, Mosquera (Cundinamarca-Colombia). I. Riqueza y estructura. Caldasia, 28(2), 243-257.

Porras, S. (2011). Diversidad y abundancia de los musgos (Briophyta) en un gradiente altitudinal del Parque Natural Municipal Robledales de Tipacoque (Boyacá-Colombia). (Trabajo de grado). Universidad Pedagógica y Tecnológica de Colombia, Boyacá, Colombia.

Richards, P. W. (1984). The ecology of tropical forest bryophytes. In R. M. Schuster (Ed.), New manual of bryology (Vol. 2, pp. 1233-1270). Nichinan: Hattori Botanical Laboratory.

Romero, C., Puts, F., \& Kitajima, K. (2006). Ecophysiology in relation to exposure of pendant epiphytic bryophytes in the canopy of a tropical montane oak forest. Biotropica, 38(1), 35-41.

Sharp, A. J., Crum, H., \& Eckel, P. M. (1994). The moss flora of Mexico. (Parts one and two). Memoirs of New York Botanical Garden, 69, 943-945.

Sillett, S. \& Antonie, M. (2004). Lichens and bryophytes in forest canopies. In M. Lowman \& H. Rinker (Eds.), Forest canopies. (2nd ed., pp.151-174). San Diego, California: Elservier Academic Press.

Simijaca, D. (2011). Líquenes epifitos de Quercus humboldtii en el Parque Natural Municipal Robledales de Tipacoque (Boyacá-Colombia). (Trabajo de grado). Universidad Pedagógica y Tecnológica de Colombia, Boyacá, Colombia.

Uribe, J. \& Aguirre, J. (1997). Clave para los géneros de hepáticas de Colombia. Caldasia, 19(1-2), 13-27.

Uribe, J. \& Gradstein, S. R. (1999). Estado del conocimiento de la flora de hepáticas de Colombia. Revista de la Academia Colombiana de Ciencias Exactas, Físicas y Naturales, 23(87), 315-318.

Van der Hammen, T. (2000). Aspectos de historia y ecología de la diversidad norandina y amazónica. Revista 
de la Academia Colombiana de Ciencias Exactas, Físicas y Naturales, 24(91), 231-245.

Vanderpoorten, A., Engels, P., \& Sotiaux, A. (2004). Trends in diversity and abundance of obligate epiphytic bryophytes in a highly managed landscape. Ecography, 27, 567-576.

Vann Leerdam, A., Zagt, R. J., \& Veenklaas, J. (1990). The distribution of epiphyte grown-forms in the canopy of a Colombian cloud-forest. Vegetatio, 87, 59-71.

Vargas, L. (2011). Hepáticas del Parque Natural Municipal Robledales de Tipacoque (Boyacá-Colombia) (Trabajo de grado). Universidad Pedagógica y Tecnológica de Colombia, Boyacá, Colombia.
Wolf, J. H. D. (1993). Diversity patterns and biomass of epiphytic bryophytes and lichens along an altitudinal gradient in the Northern Andes. Annals of Missouri Botanical Garden, 80(4), 928-960.

WOLF, J. H. D. (1995). Non-vascular epiphyte diversity patterns in the canopy of an upper montane rain forest (2550-3670 m), Central cordillera, Colombia. Selbyana, 16(2), 185-195.

Wolf, J. H. D. (2003). Estudios en ecosistemas tropandinos. Diversidad y ecología de las comunidades epifiticas en la cordillera Central, Colombia (Vol. 5, pp. 452-502). 
